\title{
Kompetenzdiagnose: Modelle, Instrumente und Tools
}

\author{
Simone Kauffeld ${ }^{1} \cdot$ Niclas Schaper ${ }^{2}$ \\ Online publiziert: 30. Juni 2021 \\ (c) Der/die Autor(en) 2021
}

Die Erfassung, Messung oder Diagnose von Kompetenzen im Rahmen organisationaler Personalentwicklungsoder anderer HRM-Prozesse wird in zunehmendem Maße bedeutsam. Entsprechende Anforderungen der Erfassung und Diagnose stellen sich unter anderem im Rahmen des organisationalen Kompetenzmanagements, d.h. der systematischen Erfassung, Entwicklung und Evaluation von Personalentwicklungsmaßnahmen, des Performance Managements und des Potenzial- bzw. Talentmanagements (vgl. Kauffeld und Paulsen 2018). Die Einführung neuer Technologien und die Veränderung von Arbeitsabläufen stellen neue Kompetenzanforderungen an Beschäftigte (Kauffeld und Maier 2020). Daher bedarf es Instrumente, die die Beschaffenheit und den Entwicklungsstand von Kompetenzen in relevanten Anforderungsbereichen organisationaler Tätigkeiten verlässlich und valide identifizieren und beschreiben können, um darauf aufbauend Beschäftigte für die Transformation weiterzuentwickeln oder auch in neue Bereiche hinein zu entwickeln. Dabei geht es insbesondere darum, eine über die Berufsausbildung hinausgehende, kontinuierliche Weiterentwicklung und Qualifizierung von Fach- und Führungskräften zu unterstützen. Besonderer Bedarf für die Erfassung und Diagnose von Kompetenzen in Organisationen ist insbesondere in Berufs- und Tätigkeitsfeldern gegeben, die durch technologische Veränderungen, die zunehmende Digitalisierung, die Veränderung von Geschäftsprozessen und Märkten (z.B. durch zunehmende Flexibilitätsanforderungen) sowie durch demographische Veränderungen gekennzeichnet sind. Hierbei gilt es, die durch zunehmend vernetzte sowie flexiblere und komplexere Prozesse neu entstehenden Kompetenzanforderungen

Univ.-Prof. Dr. Simone Kauffeld

s.kauffeld@tu-braunschweig.de

1 Arbeits-, Organisations- und Sozialpsychologie, Institut für Psychologie, TU Braunschweig, Spielmannstr. 19, 38106 Braunschweig, Deutschland

2 Institut für Humanwissenschaften, Lehrstuhl für Arbeits- und Organisationspsychologie, Universität Paderborn, Warburger Straße 100, 33098 Paderborn, Deutschland
(z.B. in Schnittstellenfunktionen von Geschäftsbereichen) valide und präzise abzubilden (vgl. z.B. Claus \& Wiese sowie Beiner, Trabert, Kinkel, Müller, Cherubini \& Lehmann, in diesem Heft). Entsprechende Ansätze dienen darüber hinaus dazu, nicht nur Diagnosen individueller Kompetenzen vorzunehmen, sondern auch Standards für die Personalentwicklung und weiterer Personalarbeit $\mathrm{zu}$ definieren und zu implementieren sowie anforderungs- und bedarfsgerechte Konsequenzen für die Weiterentwicklung der Mitarbeiter/innen und eine ausreichende Bereitstellung entsprechender Kompetenzen abzuleiten (vgl. z. B. Blumberg \& Kauffeld, in diesem Heft). So geht es auch darum, zukünftig benötigte Kompetenzen für Beschäftigte (z.B. Kinkel et al., in diesem Heft) sowie mögliche Wege der Kompetenzentwicklung innerhalb und außerhalb der Organisation, die das Potenzial der Digitalisierung nutzen, zu identifizieren (vgl. auch Kauffeld und Albrecht 2021).

Das Spektrum möglicher Ansätze der Kompetenzerfassung und -diagnose bezieht unterschiedliche Betrachtungsebenen bzw. -kriterien mit ein, die im Hinblick auf eine breite Darstellung innovativer Entwicklungen in diesem Feld abgebildet und diskutiert werden sollen: Der Fokus der Kompetenzerfassung kann auf ein breites berufliches Einsatzfeld (z.B. vgl. für handwerkliche Berufe z.B. Kauffeld und Paulsen 2018; Trainer, Coaches; vgl. Grohmann et al. 2021 oder Wißhak, Bonnes \& Hochholdinger, in diesem Heft) aber auch spezifische Tätigkeitsfunktionen gesetzt werden, die nicht nur fachspezifisch (z.B. Diagnose von technischen Störungen) sondern auch fachübergreifend definiert sind wie z. B. interkulturelle Kompetenzen, Medienkompetenzen, Schnittstellenkompetenzen, oder Führungskompetenzen (vgl. Dörr, Schmidt-Huber \& Maier, in diesem Heft) oder besondere Kompetenzgrade/-ausprägungen (z.B. Expertenkompetenzen in der Produktion) betreffen. Neben der Kompetenzerfassung bei Personen sind auch Ansätze zur Erfassung team- oder organisationsbezogener Kompetenzen von Interesse (vgl. z. B. Kauffeld 2006; Claus $\&$ Wiese, in diesem Heft).

Hinsichtlich der methodischen Konzeption werden sowohl Ansätze, die auf Selbst- oder Fremdbewertun- 
gen (z.B. Kauffeld, in diesem Heft) beruhen, als auch (Leistungs-)Testformate (z. B. Herbein, Ruth, Lipphardt \& Ringeisen, in diesem Heft), die u.a. neue mediale Formate wie z.B. Vignetten oder Simulationen einsetzen, einbezogen (vgl. Claus \& Wiese, in diesem Heft). Darüber hinaus ist auch die Darstellung der informationstechnischen Handhabung bei der Erfassung, Auswertung und Ergebnisdarstellung von Kompetenzen (z. B. als Datenbankgestützte Anwendung oder als Online-Plattform) sowie die Berücksichtigung von zielgruppenspezifischen Nutzungsvoraussetzungen/-präferenzen und Anwendungszwecken im Sinne einer zunehmend geforderten Praxis- und Nutzerorientierung von besonderem Interesse (vgl. z.B. das Kompetenznavi von Kauffeld und Paulsen 2018; Kortsch et al. 2018 oder Decius \& Schaper, in diesem Heft). Wie datengetriebene Ansätze (Big Data und Predictive Analytics) zur Kompetenzdiagnose genutzt werden können (z.B. aufbauend auf Patentanalysen) haben Karwehl und Kauffeld (2021) aufgezeigt.

Vor dem Hintergrund der skizzierten Themen, Problemstellungen und systematischen Kriterien sind daher Beiträge zur Entwicklung innovativer Kompetenzdiagnoseinstrumente bzw. -ansätze von Interesse gewesen, die (1) auf einem Kompetenzmodell bzw. einer theoretisch und/oder empirisch fundierten Anforderungsermittlung und -modellierung beruhen, (2) definierte Zielsetzungen der Kompetenzmessung und -entwicklung mit Bezug auf einen bestimmten Anwendungskontext verfolgen, (3) unabhängig gegenüber der Art des Kompetenzerwerbs sind (z. B. in dem nur Kompetenzfacetten erfasst werden, die durch einen bestimmten Trainingsansatzes adressiert werden), (4) Aussagen zu psychometrischen Gütekriterien (Objektivität, Reliabilität, Validität) treffen bzw. Belege für deren Überprüfung aufweisen und (5) auch im Hinblick auf Kriterien der Handhabbarkeit, Ökonomie und Akzeptanz der Messung gestaltet worden sind (vgl. auch Kauffeld 2006; Kauffeld und Paulsen 2018).

Die in diesem Themenheft präsentierten Beiträge lassen sich im Hinblick auf drei Bereiche gliedern: (Prospektive) Erhebung zukunftsorientierter Kompetenzen, Erfassung umfangreicher Kompetenzmodelle und Messung spezifischer Kompetenzfacetten.

Auf zukunftsorientierte Kompetenzen und ihre (prospektive) Erhebung haben sich u. a. Blumberg und Kauffeld in der industriellen Fertigung konzentriert. Sie stellen in ihrem Beitrag dar, wie digitale Werkerassistenzsysteme, z. B. Datenbrillen und Smartwatches, Mitarbeiter*innen in der Fertigung mit kontextsensitiven Informationen unterstützen und gleichzeitig neue Kompetenzanforderungen an Beschäftigte stellen. Zukünftig benötigte Kompetenzen für Beschäftigte in der Produktion sowie mögliche Wege der Kompetenzentwicklung werden anhand von 76 halbstrukturierten Interviews mit Wissenschaftlern, politischen
Akteuren und Praktikern aus dem betrieblichen Kontext identifiziert, die neben Fach-, Methoden-, Sozial- und Selbstkompetenz die digitale Kompetenz (v.a. die IT Anwendungskompetenz) als Querschnittskompetenz betont. Zur Kompetenzentwicklung wird v.a. auf selbstinitiierte Lernformate verwiesen, die Möglichkeiten des digitalisierten Lernens nutzen.

Der digitale Wandel und veränderte Kundenbedürfnisse zwingen Unternehmen zunehmend dazu, hybride Produkt-Service-Systeme zu entwickeln, wodurch spezifische Anforderungen an die Kompetenzen der Belegschaft gestellt werden. Im Beitrag von Beiner, Trabert, Kinkel, Müller, Cherubini und Lehmann werden Kompetenzen für geschäftsmodellentwickelnde Teams in traditionellen Industrieunternehmen identifiziert. Darüber hinaus werden Anregungen gegeben, welche Lehr- und Lernangebote geeignet sind, diese Kompetenzen zu fördern.

Kato-Beiderwieden, Ötting, Schlicher, Heppner und Maier (in diesem Heft) stellen die Prospektive Kompetenzanalyse (ProKA), die in mehreren Praxisprojekten erprobt wurde, vor. Dieses Instrumentarium wurde mit dem Ziel entwickelt, zukünftig erforderliche Kompetenzen für die erfolgreiche Ausführung einer beruflichen Tätigkeit prospektiv zu erfassen und die Auswirkungen anstehender organisationaler Veränderungen proaktiv in die Personalentwicklung einbeziehen zu können. Die Prospektive Kompetenzanalyse gliedert sich in drei Phasen: Zunächst werden die derzeit zur Tätigkeitsausübung erforderlichen Kompetenzen erfasst und in ihrer Wichtigkeit bewertet, um eine Vergleichsbasis zu erhalten. Anschließend wird mit verschiedenen Techniken eine Vision der zukünftigen Arbeitssituation erarbeitet und prospektiv in einer Vignette beschrieben. Auf dieser Basis wird bewertet, welche Kompetenzen zukünftig benötigt werden. Abschließend werden die Wichtigkeitseinstufungen der derzeitigen und zukünftigen Kompetenzen miteinander verglichen, um auf dieser Grundlage anforderungsgerechte Maßnahmen für die Qualifizierung der Beschäftigten abzuleiten.

Decius und Schaper illustrieren die Implementierung und Anwendung eines Tools für die strategische Kompetenzentwicklung - die Kompetenzmanagementtabelle (KMT) anhand eines betrieblichen Fallbeispiels in einem KMU. Dabei werden sowohl der Ist-Stand an vorhandenen fachlichen, interpersonellen, methodischen und kenntnisbezogenen Kompetenzen sowie sprachliche und außerberufliche Kompetenzen als auch der Vergleich von aktuellen mit zukünftigen Tätigkeitsanforderungen in den Blick genommen. Kriterien der einfachen Handhabbarkeit, der zielgruppenorientierten Verständlichkeit sowie der Umsetzbarkeit als datenbankgestütztes System bzw. der Anschlussfähigkeit an bestehende Systeme wurden bei der Entwicklung berücksichtigt, um Personalentwicklungs- und Führungskräfte in 
kleinen und mittleren Unternehmen das strategische Kompetenzmanagement zu erleichtern.

Interdisziplinäre Zusammenarbeit gilt als zukunftsweisend, um Innovationen an den Schnittstellen zwischen etablierten Disziplinen zu erreichen, wie beispielsweise im System Engineering (Huth und Vietor 2020). Claus und Wiese stellen die qualitativ-induktive Entwicklung eines Modells interdisziplinärer Kompetenzen mit den Dimensionen: Initiative zum Austausch, Zielgruppenspezifische Kommunikation, Wissensintegration und Reflexion der eigenen Fachdisziplin, vor. Zur Erfassung wurde eine Skala für das Selbstkonzept interdisziplinärer Kompetenzen, die auf dem Modell beruht, sowie einen simulationsorientierten Zugang, der zwei Aufgabenszenarien zur Verhaltenssimulation und ein biographisches Interview zur Beschreibung und Reflexion eigenen vergangenen Verhaltens in interdisziplinären Situationen beinhaltet und ebenfalls die vier Dimensionen erfasst, entwickelt und erprobt.

Mit der Modellierung und Erfassung umfangreicher bzw. erschöpfender Kompetenzmodelle für bestimmte Anforderungsbereiche befassen sich verschiedene Beiträge in diesem Heft. Kauffeld stellt mit dem Kompetenz-ReflexionsInventar (KRI), das hinsichtlich zentraler Gütekriterien an Berufstätigen überprüft wurde, eine Möglichkeit der standardisierten und ökonomischen Erfassung des Selbstkonzepts beruflicher Kompetenz vor. Dabei werden neben fachlichen (z. B. Konzeptionelles Denken, Kenntnis der Organisation und Abläufe, Problemerkennung, Entwicklung von Lösungen) methodische (z.B. Moderation und Präsentation, Planung, Reflexivität, Umgang mit EDV und Technik, Konzentration, Aufgabenverteilung) und soziale (z.B. Positionierung des eigenen Standpunktes, Motivation von Anderen, Akzeptanz und Anerkennung, Kontaktaufbau und -pflege, Rücksichtnahme und Besonnenheit) Kompetenzen berücksichtigt. Für die Selbstkompetenz wurden die Skalen Mitwirkung, Verantwortungsübernahme und Initiative, Veränderungsinteresse sowie Selbstmanagement identifiziert.

Muschalla nimmt überfachliche Kompetenzen in den Blick und beschreibt in ihrem Beitrag ein etabliertes Standardkonzept zur fähigkeitsorientierten Arbeitsanforderungs- und Arbeitsfähigkeitsbeschreibung, welches sich im Rahmen der Arbeitsfähigkeitsbegutachtung bewährt hat. Die 13 Fähigkeitsdimensionen (1) Fähigkeit zur Anpassung an Regeln und Routinen, (2) Fähigkeit zur Planung und Strukturierung von Aufgaben, (3) Flexibilität und Umstellungsfähigkeit, (4) Kompetenz- und Wissensanwendung, (5) Entscheidungs- und Urteilsfähigkeit, (6) Proaktivität und Spontanaktivitäten, (7) Widerstands- und Durchhaltefähigkeit, (8) Selbstbehauptungsfähigkeit, (9) Konversation und Kontaktfähigkeit zu Dritten, (10) Gruppenfähigkeit, (11) Fähigkeit zu engen dyadischen Beziehungen, (12) Fähigkeit zur Selbstpflege und Selbstversorgung, und (13) Mobilität und Verkehrsfähigkeit. ermöglichen einen
Abgleich des Fähigkeitsprofils der Person mit dem Anforderungsprofil des Arbeitsplatzes. Neben dem FähigkeitenFremdrating werden eine Fähigkeiten-Selbstratingversion (Mini-ICF-APP-S), und eine Version zur fähigkeitsorientierten Arbeitsplatzbeschreibung (Mini-ICF-APP-W) für die Herstellung passender Arbeitsplätze vorgestellt.

Dörr, Schmidt-Huber und Maier stellen mit dem LEaDModell (Leadership Effectiveness and Development) ein theoriebasiertes und zugleich praxisrelevantes Kompetenzmodell für wirksame Führung, das empirisch validiert wurde, vor. Mit dem Instrument werden folgende Führungskompetenzen erfasst: Strategien entwickeln, Ergebnisse erzielen, Mitarbeiter und Teams fördern, Umfeld gestalten, Persönliche Einflussnahme. Einsatzbereiche werden sowohl auf individueller (z.B. als Führungsfeedback mit Selbstund Fremdeinschätzung) als auch organisationaler Ebene (z.B. Messung der Führungseffektivität und Entwicklung der Führungskultur) gesehen. Mit dem LEaD-Modell soll darüber hinaus die Umsetzung aktueller HR-Konzepte wie das „,beidhändige Führen im Kontext der digitalen Transformation“ und eine ,entwicklungsorientierte Diagnostik“ unterstützt werden.

Mit dem Kompetenzinventar Profifußball (KIPF) stellt Decius ein Fragebogeninstrument vor, um die beruflichen Handlungskompetenzen bei Profifußballspielern anhand von Selbsteinschätzungsitems zu erfassen. Das KIPF basiert auf einem Kompetenzstrukturmodell, dessen Entwicklung anhand einer umfangreichen Literaturrecherche und auf Grundlage von Experteninterviews vorgenommen wurde. Die finale, psychometrische überprüfte Version des KIPF umfasst die acht Kompetenzdimensionen: Taktisches Wissen, Wissen zu Ernährung und Gesundheit, Einsatz von Resilienzmethoden, Umgang mit Medien, Kommunikationsfähigkeit, Teamunterstützende Anpassungsfähigkeit, Mentale Stärke sowie Kritikfähigkeit. Neben der Überprüfung der dimensionalen Struktur des Instruments wurden außerdem verschiedene konstruktbezogene Validitätsannahmen untersucht und weitgehend bestätigt. Das Inventar kann nicht nur zur Bilanzierung eigener Stärken und Schwächen, sondern auch von weiteren Anwendern (z. B. Trainer, Spielerberater) genutzt werden, um nach der Kompetenzdiagnose eine Förderung der vorhandenen Stärken und einen Ausgleich der Schwächen bei den Fußballspielern zu unterstützen.

Auf die Kompetenzmodellierung und -erfassung spezifischer Kompetenzfacetten (z.B. Präsentationskompetenz oder pädagogische Kompetenz oder Wissen) insbesondere bei einzelnen Berufsgruppen konzentrieren sich die folgenden Beiträge:

Wißhak, Bonnes und Hochholdinger stellen ein Selbstund Fremdbewertungsinstrument für die Ermittlung des Handlungswissens von Trainern, d.h. Personen, die berufliche und betriebliche Fort- und Weiterbildungsangebote 
durchführen, vor. Mithilfe des Instruments werden 41 Wissensfacetten, die sich auf 10 übergeordnete, theoretisch fundierte Wissensdimensionen verteilen (z. B. Wissen über die Planung und Konzeption von Trainings Kenntnisse über Gruppenmanagement, die methodische Umsetzung von Trainings, Diagnostik, bildungswissenschaftliche Grundlagen, individuelle Unterschiede der Teilnehmenden, Fachwissen, fachdidaktisches Wissen, Organisationswissen sowie Kenntnisse über Beratung und Coaching), erfasst. Das Instrument ProwisT kann von Trainer/innen zur Bilanzierung des eigenen professionellen Wissens und damit als Ausgangsbasis für eine individualisierte Kompetenzentwicklung genutzt werden, ebenso wie von Trainingsanbietern oder Trainerausbildungsinstituten zur individuumsübergreifenden Bedarfsermittlung (vgl. auch Grohmann et al. 2021 für ein Trainerkompetenzinventar).

Schürmann, Bender und Grebe haben ein Instrument zur Diagnose pädagogischer Kompetenzen von Pflegelehrpersonen (PädKomPflege) entwickelt und erprobt. Die entsprechenden Kompetenzen werden auf der Basis von Selbsteinschätzungen erhoben. Das zugrunde liegende Kompetenzmodell und die Befragungsitems wurden auf der Grundlage von Empfehlungen der Kultusministerkonferenz zu den bildungswissenschaftlichen Standards für die (allgemeine) Lehrerbildung sowie Expertenworkshops und -interviews mit PflegedidaktikerInnen entwickelt. Das Instrument umfasst 54 Items in fünf Hauptskalen und 18 Subskalen und erfüllt sowohl klassische als auch probabilistische Testgütekriterien. Es kann nach Angabe der Autor*innen begleitend zu Qualifizierungsprozessen als Monitoring-Instrument oder zur individuellen Kompetenzbilanzierung eingesetzt werden.

Herbein, Ruth, Lipphardt und Ringeisen konzentrieren sich auf die Konzeptualisierung von Präsentationskompetenz, d.h. einer Facette kommunikativer Kompetenzen, die in vielen Arbeitsbereichen und unterschiedlichen Branchen genutzt wird, um Wissen und Informationen weiterzugeben. Die Autoren bieten in ihrem Beitrag einen Überblick zu validierten deutschsprachigen Selbst- und Fremdeinschätzungs-Instrumenten zur Erfassung von Präsentationskompetenz. Darauf aufbauend wird ein konzeptioneller, disziplinübergreifender Rahmen vorgestellt, der insbesondere auf psychologischen und rhetorischen Theorien und Modellen basiert. Vor diesem Hintergrund werden schließlich Implikationen zur spezifischen Erfassung und bedarfsorientierten Förderung von Präsentationskompetenz, u.a. mit Bezug auf eigene Instrumente und Fördermaßnahmen, entwickelt.

Zusammenfassend werden in dem Heft verschiedene Kompetenzdiagnoseinstrumente vorgestellt, die die Breite und Heterogenität der Forschungs- und Entwicklungsansätze bei diagnostischen Instrumenten zur Kompetenzerfassung für personalwirtschaftliche und berufliche
Anwendungszwecke wiedergeben. Aus einer inhaltlichen Perspektive werden sowohl Ansätze, die auf eine sehr breite Erfassung von Kompetenzen gerichtet sind, als auch Konzepte, die sich auf spezifische Kompetenzfacetten fokussieren. Zunehmend von Bedeutung, insbesondere im Kontext der Kompetenzerfassung bei digitalen Transformationen, sind auch Ansätze einer prospektiven Kompetenzermittlung. Vielfältig sind daher die Problem- und Fragestellungen, die mithilfe der Modelle und Instrumente bearbeitet werden sollen, und vor diesem Hintergrund die konzeptionell-theoretischen Zugänge und das Vorgehen bei der Konstruktion des Instrumentariums prägen. Überwiegend wird dabei ein kombiniertes sowohl deduktiv (d.h. auf bereits vorhandenen theoretischen Konzepten oder Standards beruhendes) als auch induktiv (d.h. empirisch fundiertes) ausgerichtetes Vorgehen bei der Modellund Instrumentenentwicklung gewählt. Die methodischen Zugänge zur Erfassung von Kompetenzen reichen dabei von Selbst- und Fremdeinschätzungsinstrumenten bis hin zu testorientierten, simulativen Zugängen in Form von Verhaltenssimulationen oder prospektiven Anforderungsszenarien. Es wird darüber hinaus eingegangen auf die Überprüfung von Gütekriterien des Instrumentariums (Objektivität, Reliabilität, Validität, Handhabbarkeit, Ökonomie, Akzeptanz etc.), wobei in diesem Zusammenhang noch erheblicher Forschungs- und Überprüfungsbedarf bei einzelnen Instrumenten besteht. Schließlich werden auch der praktische Einsatz- bzw. Nutzungsmöglichkeiten der Instrumentarien dargestellt, der überwiegend im Bereich der Bilanzierung von Kompetenzen und der Ableitung von Entwicklungs- bzw. Fördermaßnahmen besteht und weniger im Bereich selektiver und bewertender diagnostischer Zwecke. Nicht zuletzt werden auch die Limitationen der einzelnen Instrumentarien diskutiert und ein Ausblick auf weiteren Entwicklungsbedarf gegeben. Dabei stellen sich auch Fragen, wie zukunftsbezogene Kompetenzen in die Modelle und Messinstrumente integriert werden können und welchen Beitrag Ansätze, die auf künstliche Intelligenz zurückgreifen, für die Aktualisierung und den Zukunftsfokus leisten können (vgl. Karwehl und Kauffeld 2021). Mit dem Fokus auf der Kompetenzdiagnose baut das Heft auf dem Vorgängerheft in der Gruppe-OrganisationInteraktion auf, mit dem das Kompetenzmanagement als Ganzes in den Blick genommen wurde (Kauffeld und Allbrecht 2021) und vertieft einen existentiellen Bestandteil: die Kompetenzmodellierung und -diagnose.

Das Heft wird in seinem diagnostischen Schwerpunkt abgerundet durch einen Beitrag zur Entwicklung und ersten Validierung eines Fragebogens zu Ressourcen und Anforderungen (ReA) in der Arbeitswelt (Schulte, Wittner und Kauffeld, in diesem Heft). Es wird ein branchenunabhängiges Analyseinstrument zur Erfassung von Anforderungen und Ressourcen aufbauend auf dem Job Demands- 
Resources (JDR) Modell und einer umfassenden Literaturrecherche in Organisationen vorgestellt. Aufbauend auf dem Einsatz des Instrumentes, der zur Gesundheitsprävention und im Rahmen der Gefährdungsbeurteilung zum Einsatz kommen könnte, können Maßnahmen zur Reduktion der Anforderungen sowie zur Stärkung der Ressourcen von Mitarbeitenden abgeleitet werden.

Abschließend bietet Kühl (in diesem Heft) eine systemtheoretisch informierte Unterscheidung zwischen Teams, Cliquen und Gruppen an. Unterschiede der drei Systemtypen werden im Hinblick auf Mitgliedschaft, Hierarchien und Zwecken aufgezeigt sowie der Nutzen der Differenzierung für Forschung und Praxis aufgezeigt.

Funding Open Access funding enabled and organized by Projekt DEAL.

Open Access Dieser Artikel wird unter der Creative Commons Namensnennung 4.0 International Lizenz veröffentlicht, welche die Nutzung, Vervielfältigung, Bearbeitung, Verbreitung und Wiedergabe in jeglichem Medium und Format erlaubt, sofern Sie den/die ursprünglichen Autor(en) und die Quelle ordnungsgemäß nennen, einen Link zur Creative Commons Lizenz beifügen und angeben, ob Änderungen vorgenommen wurden.

Die in diesem Artikel enthaltenen Bilder und sonstiges Drittmaterial unterliegen ebenfalls der genannten Creative Commons Lizenz, sofern sich aus der Abbildungslegende nichts anderes ergibt. Sofern das betreffende Material nicht unter der genannten Creative Commons Lizenz steht und die betreffende Handlung nicht nach gesetzlichen Vorschriften erlaubt ist, ist für die oben aufgeführten Weiterverwendungen des Materials die Einwilligung des jeweiligen Rechteinhabers einzuholen.

Weitere Details zur Lizenz entnehmen Sie bitte der Lizenzinformation auf http://creativecommons.org/licenses/by/4.0/deed.de.

\section{Literatur}

Grohmann, A., Schulte, E. M., \& Kauffeld, S. (2021). Entwicklung und Erprobung eines Kurzfragebogens zur Beurteilung von Trainer/ innenkompetenzen in beruflichen Weiterbildungsmaßnahmen. Gruppe. Interaktion. Organisation. Zeitschrift für Angewandte Organisationspsychologie (GIO), 52(1), 79-90. https://doi.org/ 10.1007/s11612-021-00562-0.

Huth, T. \& Vietor, T. (2020) Systems Engineering in der Produktentwicklung: Verständnis, Theorie und Praxis aus ingenieurswissenschaftlicher Sicht. Gruppe. Interaktion. Organisation. Zeitschrift für Angewandte Organisationspsychologie (GIO), 84(1), 169. https://doi.org/10.1007/s11612-020-00505-1.

Karwehl, L.J., \& Kauffeld, S. (2021). Traditional and new ways in competence management: application of HR analytics in competence management. Gruppe. Interaktion. Organisation. Zeitschrift für Angewandte Organisationspsychologie (GIO), 52(1), 7-24. https://doi.org/10.1007/s11612-021-00548-y.

Kauffeld, S. (2006). Kompetenzen messen, bewerten, entwickeln. Stuttgart: Schäffer-Poeschel.

Kauffeld, S., \& Albrecht, A. (2021). Kompetenzen und ihre Entwicklung in der Arbeitswelt von Morgen: branchenunabhängig, individualisiert, verbunden, digitalisiert? Gruppe. Interaktion. Organisation. Zeitschrift für Angewandte Organisationspsychologie (GIO), 52(1), 1-6. https://doi.org/10.1007/s11612-021-00564-y. Download frei verfügbar.
Kauffeld, S., \& Maier, G. W. (2020). Digitalisierte Arbeitswelt. Gruppe. Interaktion. Organisation. Zeitschrift für Angewandte Organisationspsychologie (GIO), 51(1), 1-4. https://doi.org/10.1007/ s11612-020-00508-y.

Kauffeld, S., \& Paulsen, H. (2018). Kompetenzmanagement in Unternehmen. Kompetenzen beschreiben, messen, entwickeln und nutzen. Stuttgart: Kohlhammer.

Kortsch, T., Paulsen, H., \& Kauffeld, S. (2018). Unterstützungskultur trifft auf digitale Lösungen: Kompetenzentwicklung mit dem KOMPETENZ-NAVI optimieren. In S. Kauffeld \& F. Frerichs (Hrsg.), Kompetenzmanagement in kleinen und mittelständischen Unternehmen: Eine Frage der Betriebskultur? (S. 181-193). Berlin: Springer.

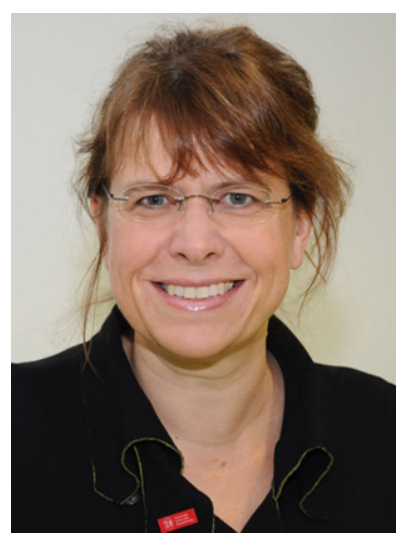

Univ.-Prof. Dr. Simone Kauffeld ist Inhaberin des Lehrstuhls für Arbeit-, Organisations- und Sozialpsychologie der Technischen Universität Braunschweig. In ihrer Forschungstätigkeit setzt sie sich in zahlreichen Projekten mit den Themen Kompetenzentwicklung und -management (Training und Transfer), Team und Führung, Karriere/ Coaching sowie Veränderungen in Organisation und Arbeit auseinander. Das Thema Digitalisierung ist als Querschnittsthema präsent. Als Herausgeberin hat sie die Zeitschriften „PersonalQUARTERLY“ und „Gruppe. Interaktion. Organisation“ neu aufgesetzt und gibt Buchreihen zur Arbeits- und Organisationspsychologie heraus. Um aktiven Wissenstransfer zu leisten, hat sie 2008 die 4 A-SIDE GmbH gegründet, die psychologische Expertise mit IT-Kompetenz verbindet.

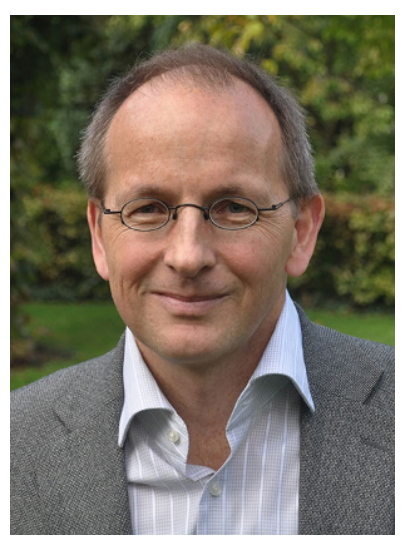

Prof. Dr. Niclas Schaper ist Professor für Arbeits- und Organisationspsychologie an der Universität Paderborn. Er hat verschiedene Forschungsprojekte zu Fragen des Kompetenzmanagements bei der strategischen Personalarbeit in KMUs und bei der Curriculum- und Personalentwicklung im Hochschulkontext geleitet. AuBerdem war er mit verschiedenen Teilprojekten an der Clusterförderung IT'sOWL zu Fragen von Industrie 4.0 und Arbeit 4.0 beteiligt. Aktuell beschäftigt er sich in einem BMBF-Projekt im Kontext der Förderlinie „Zukunft der Arbeit“ mit Fragen der partizipativen und humangerechten Begleitung von KMUs bei der digitalen Transformation von Arbeitsprozessen. Seine Forschungsschwerpunkte und -interessen liegen in den Bereichen Kompetenzerfassung und -modellierung, betriebliche Laufbahngestaltung, betriebliches Weiterbildungsverhalten insbesondere in Form von informellen Lernaktivitäten im Arbeitskontext und seine Einflussfaktoren, Erfassung organisationaler Lernkulturen, Weiterentwicklung von Arbeits- und Anforderungsanalysen, Mitarbeiterbefragungen und organisationale Bindung von Mitarbeitern. 